\title{
Medial Wedge Opening High Tibial Osteotomy with an Extra Screw Fixation
}

\author{
Ahmed A. Khalifa ${ }^{1,3, \odot}$ \\ Omar Refai ${ }^{1,2}$ \\ ${ }^{1}$ Department of Orthopaedic, Al-Rehab 2 Private Hospital, Assiut, \\ Egypt \\ 2Department of Orthopaedic and Trauma Surgery, Assiut University \\ Hospitals, Assiut, Egypt \\ ${ }^{3}$ Department of Orthopaedic and Traumatology, Qena Faculty of \\ Medicine and Its University Hospital, Qena, Egypt
}

\begin{abstract}
Address for correspondence Ahmed A. Khalifa, MD, FRCS, MSc, Department of Orthopaedic and Traumatology, Qena Faculty of Medicine and its University Hospital, South Valley University, Kilo 6 Qena-Safaga highway, Qena 83523, Egypt (e-mail: ahmed_adel0391@med.svu.edu.eg; mhosp@svu.edu.eg).
\end{abstract}

Int J Recent Surg Med Sci 2020;6:45-48

\begin{abstract}
Keywords

- high tibial osteotomy

- extra screw

- medial wedge

Medial wedge opening high tibial osteotomy (MWOHTO) is effective for managing a variety of knee conditions, varus malalignment of the knee, either secondary to osteoarthritis or residual adolescent tibia vara, are among the indications, fixation may be done using different methods (Stables, T-plate, Budu plate, and TomoFix). We are describing our technique of performing (MWOHTO) fixed with titanium T plate supplemented with an extra screw from the lateral side which we call "extra-focal" screw to obtain biplanar fixation which may add to the rotational stability at the osteotomy site.
\end{abstract}

\section{Introduction}

In 1911, Jackson et al introduced high tibial osteotomy and was propagated by Coventry in 1985 as an effective treatment for a variety of knee conditions including knee osteoarthritis with varus deformity and tibia vara without arthritis. ${ }^{1,2}$

Medial wedge opening high tibial osteotomy (MWOHTO) by correcting malalignment and offloading the medial compartment will protect against the progression of degenerative process using different fixation methods (Stables, T-plate, Budu plate, and TomoFix). ${ }^{3}$

Good patient selection, proper operative technique, and using rigid implants are among the perquisites to success, although with technique advancement, complications such as implant failure and union problems are still occurring with a reported incidence of 5.7\%.,5

We are going to describe our technique of doing MWOHTO fixed with a titanium $\mathrm{T}$ plate with the supplementation of an extra screw in another plane which we would like to call a "biplanar fixation by an extra-focal screw."

\section{Operative Procedure}

Preoperative assessment and planning were based on plain radiographs, standing anteroposterior (AP) and lateral views, the procedure is done under spinal anesthesia and no arthroscopy is done prior to the osteotomy.

\section{Surgical Technique}

The procedure is done on a radiolucent table while the patient is in supine position with a thigh tourniquet which is elevated to $150 \mathrm{~mm} \mathrm{Hg}$ above the systolic blood pressure. All patients receive $1 \mathrm{gm}$ first-generation cephalosporin $30 \mathrm{~min}$ utes before elevation of the tourniquet. Draping is done in the usual manner till the level of the tourniquet. An anterior midline incision is made starting from the lower pole of the
DOI https://doi.org/

$10.1055 / \mathrm{s}-0039-1697069$

ISSN 2455-7420.
License terms

() (1) $\Theta \circledast$ 
patella and extending approximately $3 \mathrm{~cm}$ distal to the joint line ( -Fig. 1A), care is taken to make the distal extension of the wound just medial and not exactly over the tibial tuberosity, if needed, the incision can be expanded to improve visualization or to ease plate insertion. Subperiosteal elevation of the superficial medial collateral ligament is done, then subperiosteal blunt dissection is continued around the posteromedial aspect of the tibia elevating the posterior structure from the back of the tibia till we can digitally feel the head of the fibula (-Fig. 1B), A Kirschner's wire was inserted under fluoroscopic guidance in an oblique direction from a point just above the upper border of the pes anserinus at the metaphyseal-diaphyseal junction of the medial proximal tibial cortex, aiming at the level of the tip of the fibular head ( Fig. 1C, D).

\section{Osteotomy Technique}

An ascending osteotomy is then made behind the tibial tuberosity (-Fig. 2) leaving about fourth of its thickness anteriorly or just $1.5 \mathrm{~cm}$ posterior to its anterior rim, leaving the patellar ligament attached to the distal tibial tuberosity fragment (the aim of this ascending osteotomy is to ease the movement of the proximal segment after doing the tibial osteotomy), then two Homman retractors were inserted, one just behind the patellar tendon and the other was inserted

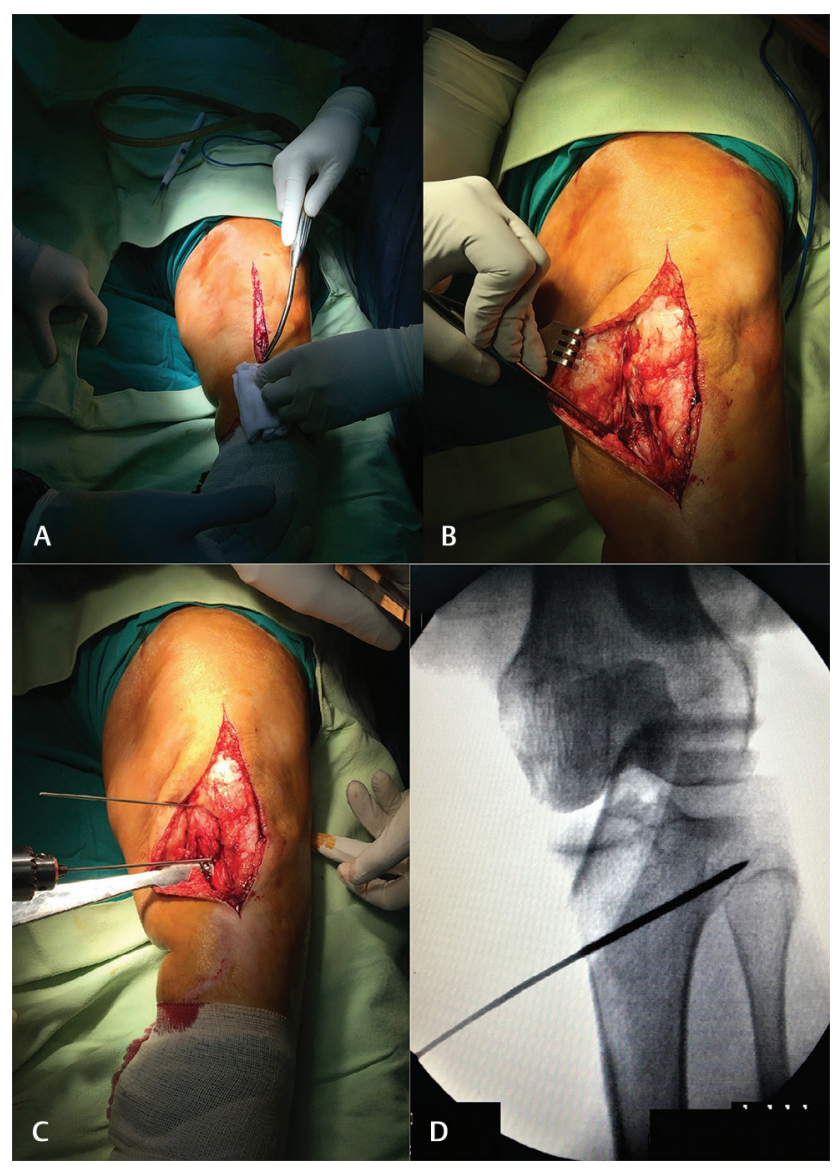

Fig. 1 (A) A mid-line incision starting from the lower pole of the patella. (B) Periosteal dissection on the medial side. (C) Placement of a Kirschner's wire aiming at the head of the fibula, starting from the proposed osteotomy site. (D) Checking the Kirschner's wire position under fluoroscopic guidance. between the posterior aspect of the tibia and the dissected medial and posterior structures. The main tibial osteotomy is then performed just beneath the wire using a sharp osteotome while the knee is flexed to 15 degrees (to keep the posterior structures relaxed and away from the osteotome; ( - Fig. 3A, B). The osteotomy should stop $5 \mathrm{~mm}$ medial to the lateral cortex. Gradual opening of the osteotomy site

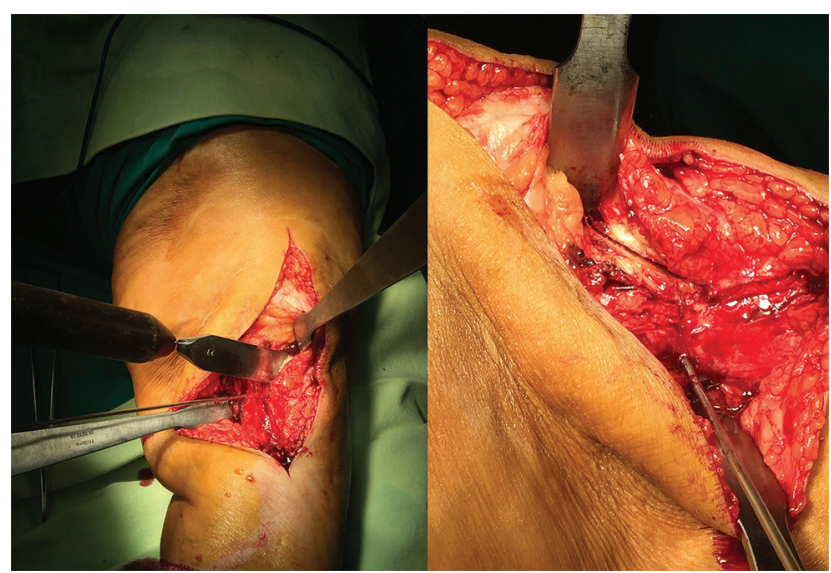

Fig. 2 Ascending osteotomy is done behind the tibial tuberosity, $1.5 \mathrm{~cm}$ posterior to its anterior rim.

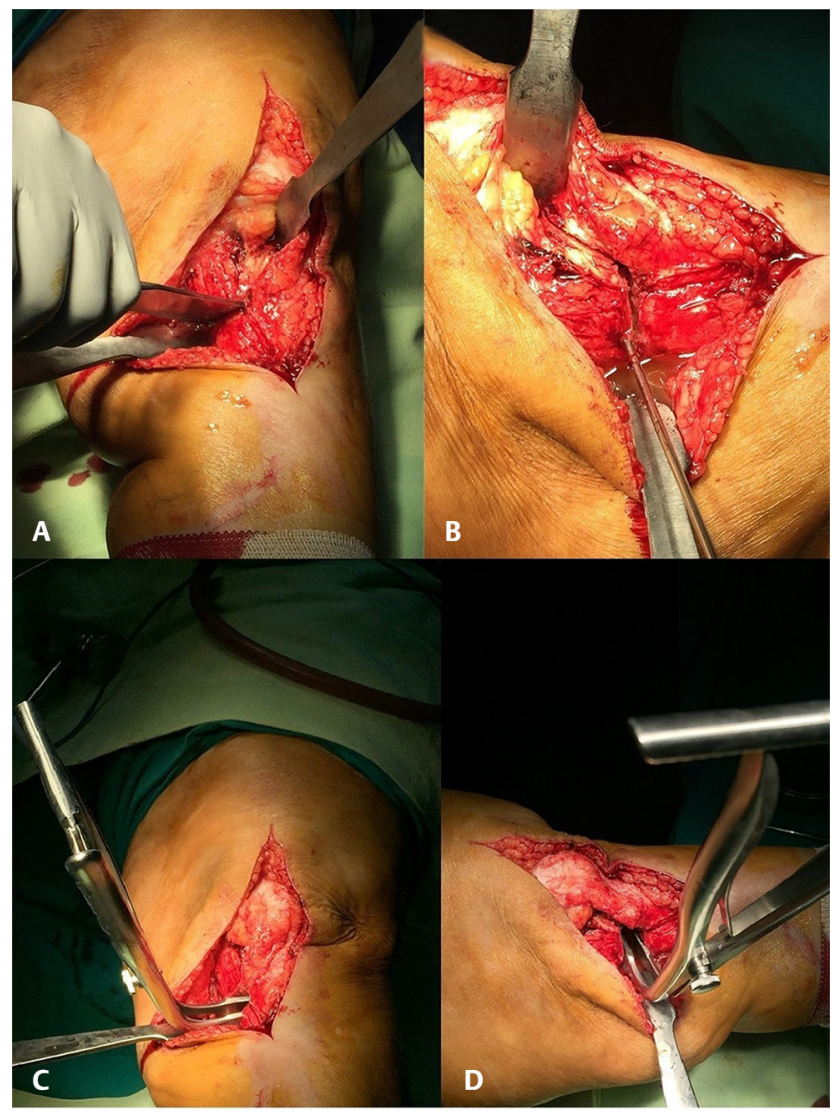

Fig. 3 (A) Placement of two Homman retractors (anterior and posterior), starting of the osteotomy using sharp osteotome just below the Kirschner's wire. (B) After finishing the osteotomy, the final view of both osteotomies (retro- tibial tuberosity and the main tibial osteotomy). (C) Placement of the laminar spreader after osteotomy opening (front view). (D) Side view shoeing amount of osteotomy opening and maintenance using laminar spreader. 
was done using two flat chisels, and the osteotomy opening is maintained using a laminar spreader ( - Fig. $\mathbf{3 C}, \mathbf{D}$ ). The intraoperative mechanical axis was assessed using the cable technique. ${ }^{6}$ with the knee in a fully extended position aiming for a mechanical axis passing at the Fujisawa's point (at $30-40 \%$ lateral to the midpoint of the tibial plateau). The aim is to obtain a slight valgus axis to prevent recurring of varus (nearly 8-10 degrees of valgus in the anatomical axis).?

$\mathrm{T}$ locked titanium plate (Orthomed-E Co., Egypt) is used to fix the osteotomy site, a nonlocked screw fixation is done first at the oval hole below osteotomy level to make sure that the plate is resting on the bone and its proper position is confirmed with fluoroscopy, then formal fixation of the remaining locked screws which is done as follows: proximal part of the plate has three locking holes for fixation in the metaphyseal area, only the two peripheral screws are secured leaving the middle screw hole empty to accommodate for the passage of the extra-focal screw ( $\boldsymbol{- F i g . ~ 4 A , ~ B ) , ~ n o ~ g r a f t ~ i s ~ n e e d - ~}$ ed, but after finishing plate fixation, with the use of a small curette, some cancellous bone is curetted from the proximal tibia through the osteotomy opening and left inside the osteotomy site as a local bone graft.

\section{Extra-Focal Screw Fixation Technique}

The same drill used for the locking screws $(4.1 \mathrm{~mm})$ is used to drill the track for this extra screw, the target is to aim the drill to a direction going from distal to proximal, starting at the level of the lateral aspect of the tibial tuberosity targeting the subchondral bone of the medial tibial condyle ( - Fig. $5 \mathbf{A}, \mathbf{B}$ ). The screw ideally should pass between the two proximal screws, the drill under fluoroscopic guide should start just at the medial plateau subchondral bone and not to penetrate it. If resistance is felt, it may be due to drill hitting either of the screws and the direction should slightly be changed, after confirming the proper position of the drill and measuring the appropriate length, a cortical screw $(5 \mathrm{~mm}$ ) from the locking screw set is used with the benefit of wider caliber than the usual cortical $4.5 \mathrm{~mm}$ screws, which theoretically should be

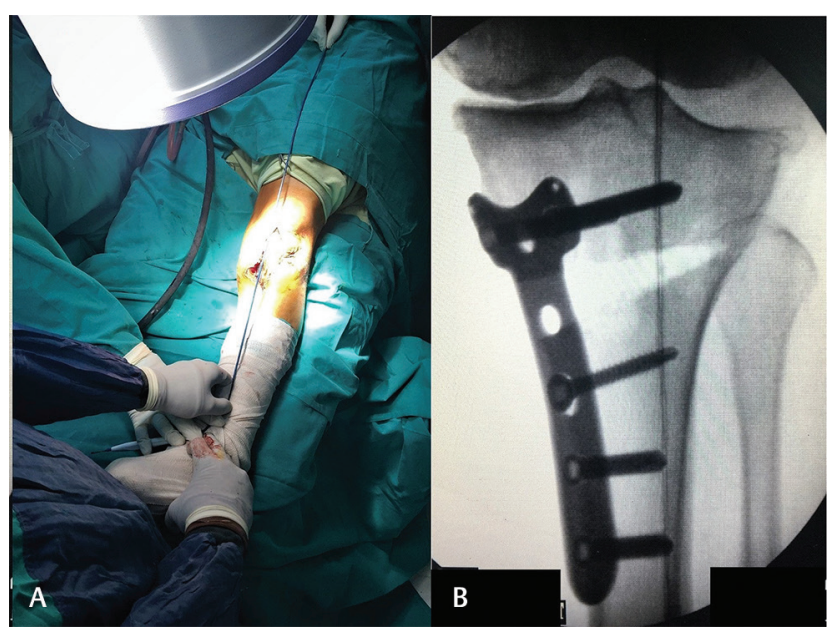

Fig. 4 (A) After plate insertion, checking the final alignment clinically using the cable technique. (B) Fluoroscopic confirmation of mechanical alignment passage through Fujisawa's point.

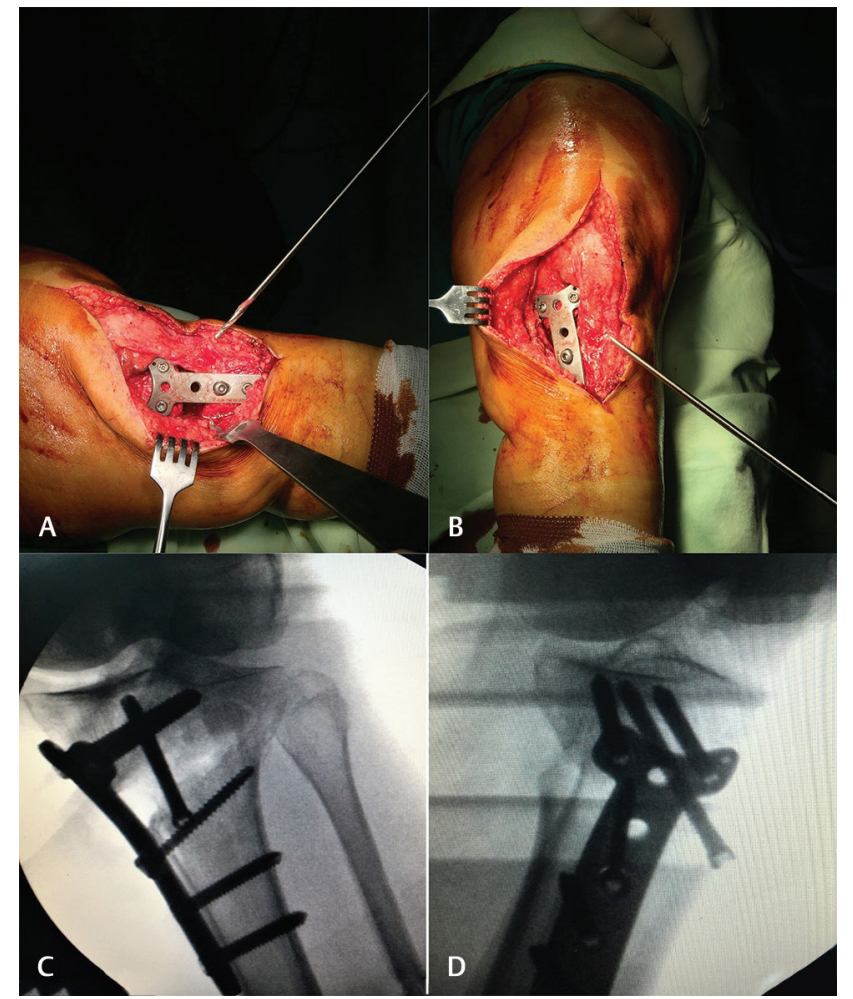

Fig. 5 Extra screw fixation. (A) Side view of the starting position of the drill (aiming from a distal to a proximal position). (B) Front view of the starting of the drill (just lateral to the tibial tuberosity, aiming toward the medial tibial plateau). (C) Anteroposterior and (D) lateral fluoroscopic views after insertion of the screw showing the optimum position of the screw and its passage between the proximal two screws in the lateral view.

more rigid. Also, the head of the screw has threads (which is designed to lock inside the hole of the plate), which may have some grip in the outer cortical shell at its entry point which we suggest that it will prevent further sinking of the screw or backing out which may cause irritation due to subcutaneous nature at this area. Care should be taken to make sure that the head of the screw is not prominent from the bone. After inserting the proper screw, the proximal construct should be formed of two screws locked to the plate and another crossing screw from the opposite side taken extra-focally from outside the plate ( - Fig. 5C, D).

Wound closed in anatomical layers without inserting a suction drain. A compressive dressing was used.

\section{Postoperative Protocol}

Isometric quadriceps exercises, active ankle pumping, and straight leg-raising exercises start on day 1 postoperative. Flexion of the knee as much as tolerated and walk with partial weight bearing as tolerated for the first 6 weeks postoperative except for heavy patients which we advised to just toe-touch weight bearing till osteotomy union is evident in the radiographs. Postoperative radiographic assessment (day 1, 6 weeks, 12 weeks, and last follow-up at 24 weeks) of a digitalized standing knee AP view (whenever possible; -Fig. 6 ). 


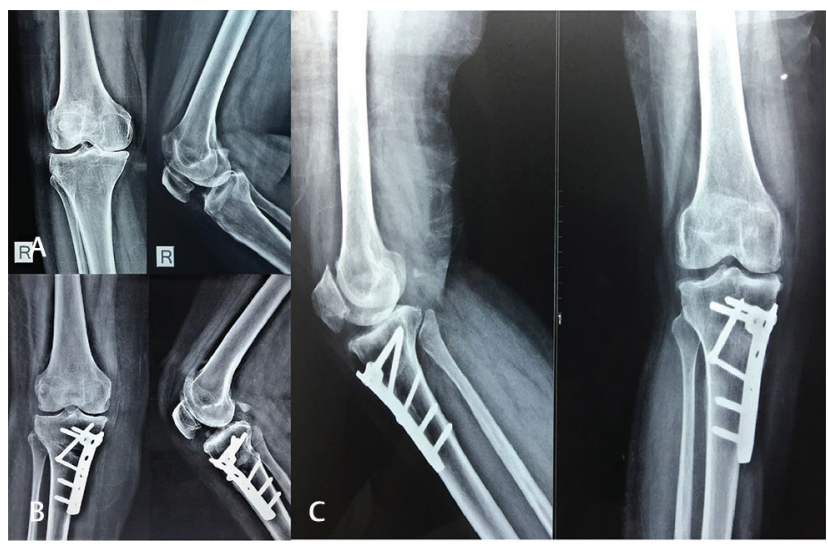

Fig. 6 Radiographic evaluation (right knee). (A) Preoperative. (B) Immediate postoperative. (C) 6 months postoperative.

\section{Discussion}

To obtain an accurate limb alignment maintained by rigid fixation are considered to be the main aim of performing MWOHTO. ${ }^{7}$ We believe that these goals could be achieved after utilizing our technique of performing the osteotomy with the supplementation of an extra fixation in another plane through the extra-focal screw.

Both biological and mechanical factors should be taken into consideration if the surgeon aims at an optimum MWOHTO outcome; lateral cortex continuity in association with strong fixation and soft tissue integrity is one among these factors. ${ }^{1,8}$

The inherent instability of the MWOHTO is due to the gap created in the proximal tibia, attention should be paid for fixation to prevent early failure with loss of correction. ${ }^{9}$

The use of locking compression plates or extra screws, as was utilized in our technique, had been recommended when the correction angle is large and a long wedge height will ensue for the fear of breaking the lateral cortex or even a tibial plateau fracture which will affect osteotomy stability. ${ }^{3}$

Intraoperative as well as postoperative complications can occur; most of these complications are caused mainly by surgical and technical errors, such as under- or overcorrection, breakage of the lateral cortex, and delayed and nonunion. ${ }^{10}$

\section{Conclusion}

We believe that adding a screw to the MWOHTO procedure is a simple, safe, and time saving technique, theoretically stiffer than fixing with the plate alone. We believe that a biomechanical study should be done to back up this theory.

\section{Note}

The initial description was presented at the Egyptian Orthopaedic Association Conference in 2017. This work had been done exclusively at Al-Rehab 2 Private Hospital.

\section{Conflict of Interest}

None declared.

\section{References}

1 Coventry MB, Ilstrup DM, Wallrichs SL. Proximal tibial osteotomy. a critical long-term study of eighty-seven cases. J Bone Joint Surg Am 1993;75(2):196-201

2 Jackson JP, Waugh W, Green JP. High tibial osteotomy for osteoarthritis of the knee. J Bone Joint Surg Br 1969;51(1):88-94

3 Lee DC, Byun SJ. High tibial osteotomy. Knee Surg Relat Res 2012;24(2):61-69

4 Chae DJ, Shetty GM, Wang KH. Montalban AS Jr, Kim JI, Nha KW. Early complications of medial opening wedge high tibial osteotomy using autologous tricortical iliac bone graft and T-plate fixation. Knee 2011;18(4):278-284

5 Seo SS, Kim OG, Seo JH, Kim DH, Kim YG, Lee IS. Complications and short-term outcomes of medial opening wedge high tibial osteotomy using a locking plate for medial osteoarthritis of the knee. Knee Surg Relat Res 2016;28(4):289-296

6 Krettek C, Miclau T, Grün O, Schandelmaier P, Tscherne H. Intraoperative control of axes, rotation and length in femoral and tibial fractures. Technical note. Injury 1998;29(Suppl 3):C29-C39

7 Sabzevari S, Ebrahimpour A, Roudi MK, Kachooei AR. High tibial osteotomy: a systematic review and current concept. Arch Bone Jt Surg 2016;4(3):204-212

8 Rose T, Imhoff AB. Complications after transgenicular osteotomies. Oper Tech Orthop 2007;17(1):80-86

9 Jackson JP, Waugh W. Tibial osteotomy for osteoarthritis of the knee. J Bone Joint Surg Br 1961;43-B(4):746-751

10 Song SJ, Bae DK, Kim KI, Lee CH. Conversion total knee arthroplasty after failed high tibial osteotomy. Knee Surg Relat Res 2016;28(2):89-98 